\title{
Traduire
}

Revue française de la traduction

$231 \mid 2014$

À table !

\section{Une plume dans le vin}

\section{Jane Maylin}

Traducteur : Hélène Ladjadj

\section{CpenEdition}

Journals

\section{Édition électronique}

URL : http://journals.openedition.org/traduire/653

DOI : $10.4000 /$ traduire. 653

ISSN : 2272-9992

Éditeur

Société française des traducteurs

Édition imprimée

Date de publication : 1 décembre 2014

Pagination : 5-14

ISSN : 0395-773X

Référence électronique

Jane Maylin, « Une plume dans le vin », Traduire [En ligne], 231 | 2014, mis en ligne le 01 décembre 2016, consulté le 02 mai 2019. URL : http://journals.openedition.org/traduire/653 ; DOI : 10.4000/ traduire.653 


\section{Une plume dans le vin}

\section{Jane Maylin}

J'ai pris le chemin de la traduction assez tardivement. J'avais choisi des études de langues par passion mais comme bien des jeunes diplômés, j'ai commencé à travailler dans un domaine qui n'avait pas vraiment de rapport avec mes études. Mais voilà, la vie nous conduit parfois en des lieux inattendus et pour moi, ce fut la France.

\section{La culture du vin}

La France - pays emblématique, pour le monde entier, de l'art de vivre, de la gastronomie et du vin. C'est inscrit dans la conscience collective : la France est le pays du bon vin, et cela malgré l'émergence de pays producteurs comme l'Argentine, le Chili ou l'Australie, qualifiés de "nouveau monde ", et l'existence d'excellents vins dans d'autres pays du vieux monde. En vérité, le vin est probablement né au Moyen-Orient et nous devons remercier les Romains et les Grecs pour sa diffusion en Occident.

Mais la France a élevé le culte du vin à un niveau inégalé avec un classement hiérarchique et un système d'appellations complexe propre à ses vins, que leur réputation prestigieuse place encore aujourd'hui parmi les plus chers et les plus recherchés au monde.

Ce véritable phénomène culturel a engendré sa propre terminologie dont certains mots sont passés dans la langue anglaise. Tout comme l'industrie aéronautique française, à l'origine des mots " aileron " et " fuselage ", les a vus repris tels quels par l'anglais, les termes " château ", " grand cru ", " appellation ", "bouteille " (transformé en bottle), " apéritif " et " sommelier " ont intégré la langue de Shakespeare. Sans oublier, bien sûr, le mot le plus symbolique de tous : " terroir ". Le concept a d'ailleurs longtemps donné du fil à retordre aux traducteurs qui ont proposé soil, situation, origin, terrain, voire territory. Mais si territoire et terroir ont une même origine étymologique, la notion de terroir est bien plus complexe.

Ce mot associe type et âge du sol (par exemple : sols typiques issus de marnes ou de calcaires durs de l'ère secondaire - grès, calcaires blancs, calcaire à gryphées, pierres dorées, plus 
quelques grands îlots volcaniques et schisteux), climat, exposition, écosystème local et végétation, plus une dimension culturelle spécifique. C'est une interaction unique de facteurs biophysiques et humains. D'ailleurs, le fait que l'UNESCO ait créé une Charte des Terroirs en dit long sur l'importance du concept(1).

Étant donné la difficulté à saisir l'essence du mot " terroir " pour l'exprimer en anglais, on a commencé à employer le mot français, (parfois avec une brève explication, en fonction du lectorat visé). D'autres mots (chiaroscuro, kindergarten, perestroika et chutzpah), venus de langues diverses, ont aussi fini par intégrer progressivement la langue anglaise selon le même processus.

Terroir est désormais accepté dans un texte anglais, même s'il est le plus souvent placé entre guillemets pour signaler qu'il ne fait pas encore tout à fait partie de la langue. Le traducteur, pour lequel certains termes sont parfaitement clairs, risque alors de croire, à tort, qu'un mot a déjà parcouru la distance entre les deux langues et peut donc être employé dans sa langue d'origine sans aucune autre explication.

\section{La traduction comme vocation}

Vocation vient du latin vocatio (appel). II faut parfois un certain temps pour identifier sa propre vocation et même emprunter bien des chemins avant de la trouver.

Vivant dans une région viticole en France, j'ai été directement impliquée dans le monde du vin pendant plus de quinze ans avec des rôles professionnels divers, allant de la commercialisation à la production. J'ai découvert l'ampélographie (la France possède la plus grande richesse d'espèces et de variétés de vignes au monde, avec 267 variétés autorisées par le ministère de l'Agriculture), et les maladies et ravageurs de la vigne. J'ai tout appris sur la réglementation concernant les traitements du vin et les mentions obligatoires sur l'étiquette, les teneurs en $\mathrm{pH}$ et en sucre résiduel, sans oublier les Documents d'accompagnement électronique. Je sais désormais qu'un groupe de vendangeurs s'appelle une colle, à ne pas confondre avec la colle agent utilisé pour la clarification du vin tels la bentonite ou le blanc d'œuf qui captent les particules en suspension, processus appelé collage, ou fining en anglais. J'ai étudié l'art de l'assemblage, commandé des matières sèches (un terme à la signification clairement énoncée : tout ce qui fait une bouteille de vin, hormis le liquide) et organisé des mises en bouteille. C'était absolument passionnant et ce fut en outre une occasion exceptionnelle d'apprendre, une source infinie d'informations fascinantes.

(1) http://www.unesco.org/new/fileadmin/MULTIMEDIA/HQ/SC/pdf/sc_mab_terroirs_FR.pdf 
Le vin est alors entré en symbiose avec mon amour des langues et, moi qui pratiquais la traduction depuis des années, j'ai su à ce moment-là que je voulais en faire mon métier à plein temps.

L'une des singularités de bien des métiers c'est l'emploi du jargon. Le monde du vin et de la viticulture ne fait pas exception:

Ébourgeonnage, levurage, piégeage, chaptalisation, cuvaison, dégorgement, délestage, éraflage, ouillage, mutage, palissage... la liste est longue.

Pour le non-spécialiste il existe des dictionnaires de référence, dont l'excellent LexiVin, mais un traducteur spécialisé doit posséder une connaissance approfondie du sujet. Armée de mon expérience professionnelle et de ma formation linguistique, je pouvais transmuer ce savoir en mots et j'ai décidé de tremper ma plume dans le vin.

\section{Le monde de la viticulture et de l'œnologie}

L'univers du vin et des spiritueux présente bien des aspects et ne se limite certes pas au liquide versé dans le verre. De la grappe au verre, chaque étape possède ses spécificités dont la traduction devra tenir compte.

Tout commence dans le vignoble, avec ses différentes méthodes de culture et de taille, les techniques de maîtrise des rendements, la panoplie des traitements phytosanitaires. C'est le domaine de la biologie et de la botanique. Le développement des techniques de viticulture biologique et biodynamique m'impose des mises à jour permanentes en tant que traductrice. Les techniques biodynamiques emploient des produits allant de la bouse de vache aux orties et aux fleurs de pissenlits et tiennent compte de l'effet des forces cosmiques sur le vivant, ouvrant ainsi à la fois un vaste champ sémantique et un débat philosophique.

Puis on passe à la cave et les choses deviennent plus techniques : analyses, mesures, traitements ; bactéries et levures, leur action sur le vin. Que se passe-t-il en cas de présence de Brettanomyces ou autres micro-organismes indésirables, comment prévenir ou lutter contre le problème ? Cet environnement a son vocabulaire propre, un lexique précis, scientifique, relevant de la chimie et de la microbiologie. II ne peut y avoir d'ambiguïté. Crushing et pressing semblent quasi équivalents en anglais, mais il existe une nuance subtile pour la vinification et il ne faut pas les confondre en traduisant car en français, foulage et pressurage sont des opérations bien distinctes nécessitant un matériel spécifique (fouloir et pressoir). Dans certains cas la traduction peut varier selon la phase du processus de vinification, ainsi marc peut être traduit par cap au stade de la fermentation (lorsque la masse de peaux de raisin flotte en surface), et devient pomace après pressurage (lorsque les peaux ont été séparées du vin). 
Des visites régulières aux caves et des contacts et discussions avec des œnologues sont donc indispensables à mon travail, tout en m'offrant d'agréables échappées loin de mon clavier !

\section{Internet et la " glouglousphère "}

II existe aussi sur internet nombre de forums et groupes bien utiles pour apporter une réponse à quelque question technique épineuse. Viticulteurs, bloggeurs, critiques et journalistes sont présents en grand nombre sur les réseaux sociaux, et cette vaste communauté du vin sur la toile est désignée par le charmant vocable de "glouglousphère ". La traduction étant une activité plutôt solitaire, ces contacts sont une ouverture bienvenue sur le monde extérieur.

II est vrai qu'internet est devenu pratiquement indispensable. Je suis sûre de ne pas être la seule à recevoir tous mes textes à traduire par courrier électronique, mais c'est surtout une source d'information inestimable pour les recherches sur le sujet que je traduis, sur l'entreprise ou le client qui m'a commandé la traduction, afin de connaître son positionnement sur le marché et donc le style requis. Cela veut dire aussi que le travail peut nous arriver du monde entier, ce qui est un avantage considérable, mais aussi une source de forte pression en termes de délais. Sur le plan pratique, je stocke mes fichiers dans le nuage (autrement dit le cloud) : ainsi, je peux y accéder où que je sois et ils sont à l'abri en cas de panne de matériel. Internet est en outre ma vitrine principale via mon site et ma présence sur les réseaux sociaux.

Mais passer le plus clair de son temps devant son écran expose à la tentation de surfer sur internet au lieu de travailler surtout que, même en déplacement, on est sollicité par son smartphone. II faut une discipline de fer pour rester concentrée et garder une approche professionnelle face aux réseaux sociaux. Me considérer comme mon propre Community Manager (animateur de communauté) m'aide toutefois à maintenir l'équilibre entre travail et distraction face au web.

\section{La veille technologique}

Revenons au travail à la cave, qui ne se limite pas à la vinification. En français, on dit joliment du vin qu'il est élevé, comme un enfant, mais en anglais il est matured ou aged (vieilli). Cela peut indiquer l'utilisation des barriques ou, dernièrement, l'introduction de morceaux de bois autorisée en France depuis 2005 seulement - pour l'élevage du vin et depuis 2009 en vinification. Pour la traduction, il faut savoir distinguer entre copeaux, barriques ou douelles, et comprendre ce que l'on entend par les différents degrés de chauffe du bois.

Pour tenir compte des évolutions techniques, il faut prendre le temps de se constituer un ensemble de connaissances que l'on maintient à jour, par exemple la mise au point de capteurs 
optiques portables pour l'évaluation de la maturité phénolique à partir de la fluorescence des raisins et qui, dans les vignobles, peut servir à déterminer les dates optimales de vendange.

Parmi les nouveautés intéressantes, citons l'emploi de cuves béton œuf : "La forme œuf induirait la création de mouvements browniens dans le liquide, dit(s) mouvement(s) "vortex", et maintiendrait les lies constamment en suspension comme un bâtonnage naturel(2) ". Passionnant!

Ou encore, l'utilisation de récipients en terre cuite (dolia) pour la vinification, des essais d'élevage de vin sous la mer, voire des tests de vieillissement des molécules de whisky dans l'espace ! Je ne me lasse jamais d'apprendre sur ces nouvelles méthodes et je suis une lectrice avide de revues sur l'œnologie et de blogs sur le vin, en français et en anglais. L'acquisition de vocabulaire et de connaissances est une quête sans fin.

\section{Adapter l'approche linguistique}

L'étape suivante est la mise en bouteilles, ou conditionnement. C'est une opération technique qui renvoie à plusieurs opérations mécaniques et à du matériel spécifique. Pour traduire un document traitant de questions techniques de ce genre, il me faut savoir quel est le niveau de connaissances du lecteur et il est donc impératif de bien communiquer avec la personne qui a commandé la traduction.

En Champagne il y a un objet très précis appelé "bidule " - oui, c'est bien ça, un machin, une chose - mais comment le traduire correctement ? Faut-il utiliser des équivalents anglais ou simplement parler d'un morceau de plastique ? Cela dépendra du contexte. Dans une présentation orale à un groupe de visiteurs, la traduction littérale provoque toujours un sourire. Un peu de folklore passe aussi sur un blog ou une lettre d'information informelle. En revanche, dans un document formel, je considère que le mot bidule est tout simplement le nom désignant cet élément. Pour ceux qui s'interrogent, il s'agit d'un opercule creux (comme un dé à coudre) en plastique, placé dans le goulot de la bouteille de champagne après ajout de la levure pour la seconde fermentation, ou prise de mousse. II sert de réceptacle pour les cellules mortes de la levure accumulées dans le goulot après remuage, et facilite leur retrait.

S'agissant du bouchage, la traduction demande de la précision. En anglais, on utilise le mot cork (bouchon) mais en français, il y a le bouchon liège et le bouchon synthétique, et même le bouchon en verre ; il faut donc qualifier le terme en anglais. Ce sera natural cork ou synthetic cork, et glass closure (glass cork étant une sorte d'oxymore - cork signifiant liège).

(2) http://www.oenologuesdefrance.fr/gestion/fichiers_publications/444_ART251_1_V.pdf 
Le bouchon de liège soulève bien sûr la question des vins bouchonnés, problème dû au trichloro-anisole ou TCA, un composé qui peut altérer le bouchon et donner au vin une odeur de carton mouillé ou de torchon sale. Pour traduire des documents évoquant ce problème il faut choisir la formulation selon le destinataire : s'il s'agit de professionnels, on veillera à l'emploi de formules chimiques et termes techniques précis, s'il s'agit de lecteurs non spécialisés, les explications devront être simples et dépourvues de jargon.

II y a un autre genre de bouchage souvent désigné par son nom anglais, screwcap, parfois préféré au français pour une bonne raison : les Français n'en sont pas très amateurs et il est plutôt associé aux vins étrangers. Bague à vis, ou capsule à vis, sont en effet des termes techniques qui n'ont pas la même tonalité. Là encore, le choix se fera en fonction du public visé.

\section{Marketing et communication}

À l'automne, les vins terminent tranquillement leur fermentation en cave. Certains vins sont déjà mis sur le marché comme primeurs, tel le Beaujolais nouveau mis en vente chaque année le troisième jeudi de novembre. Tout un rituel est né autour de ce vin dont 31 millions de bouteilles sont commercialisés dans le monde entier, et les Japonais et les Américains en sont particulièrement amateurs. Dans les pays anglophones, mais aussi dans bien d'autres parties du monde comme la Scandinavie, l'Asie et la Russie, l'anglais est la langue de prédilection d'une vaste campagne de communication, qui impose à tous les intervenants des délais stricts, y compris au traducteur.

Dans un contexte commercial, je suis souvent sollicitée pour des traductions dans des univers associés comme l'œnotourisme (et maintenant le spiritourisme dans le monde des spiritueux), et la gastronomie bien sûr, avec les accords mets-vins. Les documents à traduire vont du site web complet au communiqué de presse en passant par les brochures, notes de dégustation ou fiches techniques. Cela fait appel à des compétences dans des registres complètement différents de celui des rapports d'œnologues ou des articles techniques. Il s'agit avant tout d'outils marketing à des fins promotionnelles et le vocabulaire doit donc être choisi en conséquence.

\section{Le lexique de la perception sensorielle}

Nous en arrivons maintenant au vin dans le verre. L'un des aspects les plus fascinants du vin c'est l'immense diversité de ses arômes. II y a un rapprochement évident ici avec la gastronomie. La traduction quitte alors la sphère technique pour le monde de la perception individuelle et la problématique devient tout autre : il ne s'agit plus de trouver des définitions précises mais d'arriver à transmettre une expérience sensorielle. La mémoire olfactive est très puissante qui ne connaît le célèbre exemple de la madeleine de Proust? 
L'odeur et la saveur restent encore longtemps, comme des âmes, à se rappeler, à attendre, à espérer, sur la ruine de tout le reste, à porter sans fléchir, sur leur gouttelette presque impalpable, l'édifice immense du souvenir(3).

Un texte est déjà la traduction de ces sensations ressenties en bouche et fixées par la mémoire. La traduction dans une autre langue doit veiller à conserver l'émotion originale, mais dans un domaine aussi subjectif, la relation entre les mots et les caractéristiques décrites est plus complexe. L'objectif principal est de capter l'essence de l'expérience puisque le plaisir est un élément clé du vin. Les souvenirs olfactifs seront transmis par un choix judicieux des mots.

La fiche de dégustation française classique se présente en général dans l'ordre œil-nez-bouche et s'il faut évoquer l'aspect (couleur, limpidité, intensité), l'odeur (arômes primaire et secondaire), le goût (texture, consistance, parfum) et la persistance (caudalies), le lecteur anglophone tend à préférer un texte descriptif à une liste d'attributs.

II faut en outre s'adresser au consommateur en des termes qu'il puisse apprécier et comprendre. On sait que certaines références sont totalement incompréhensibles pour les Chinois qui n'ont jamais goûté ni même vu nombre des fruits habituellement évoqués pour parler des vins en Europe(4). Si les différences culturelles entre la France et le monde anglophone ne sont pas aussi marquées, il faut néanmoins en tenir compte.

L'homme dispose d'un grand éventail de perceptions, mais d'un nombre restreint de mots pour les décrire. Déguster du vin est une première étape pour trouver ces mots et un cours de dégustation professionnel m'a été extrêmement utile pour développer ma sensibilité à la palette des arômes et à leur position sur le spectre olfactif. Un lexique des arômes est souvent utilisé dans le secteur industriel pour décrire des produits aux fins de comparaison et de contrôle de qualité. Sivertsen et Risvik(5) ont démontré que les vins rouges français pouvaient être différenciés par région d'origine avec un lexique sensoriel. Pour les néophytes, la Roue des arômes(6) peut être un outil très utile pour trouver les correspondances au sein d'une famille d'arômes.

(3) Marcel Proust, 1987 [1913], À la recherche du temps perdu. Du côté de chez Swann, Paris, Gallimard, La Pléiade, Vol. I, p. 46.

(4) http://online.wsj.com/news/articles/SB10001424127887324735304578354481799586190

(5) Sivertsen, H.K. and Risvik, E., 1994, " A study of sample and assessor variation: A multivariate study of wine profiles ", Journal of Sensory Studies, $9: 293-312$

(6) http://www.chemins-vignerons.com/wfr/2009/02/la-roue-des-aromes/ 


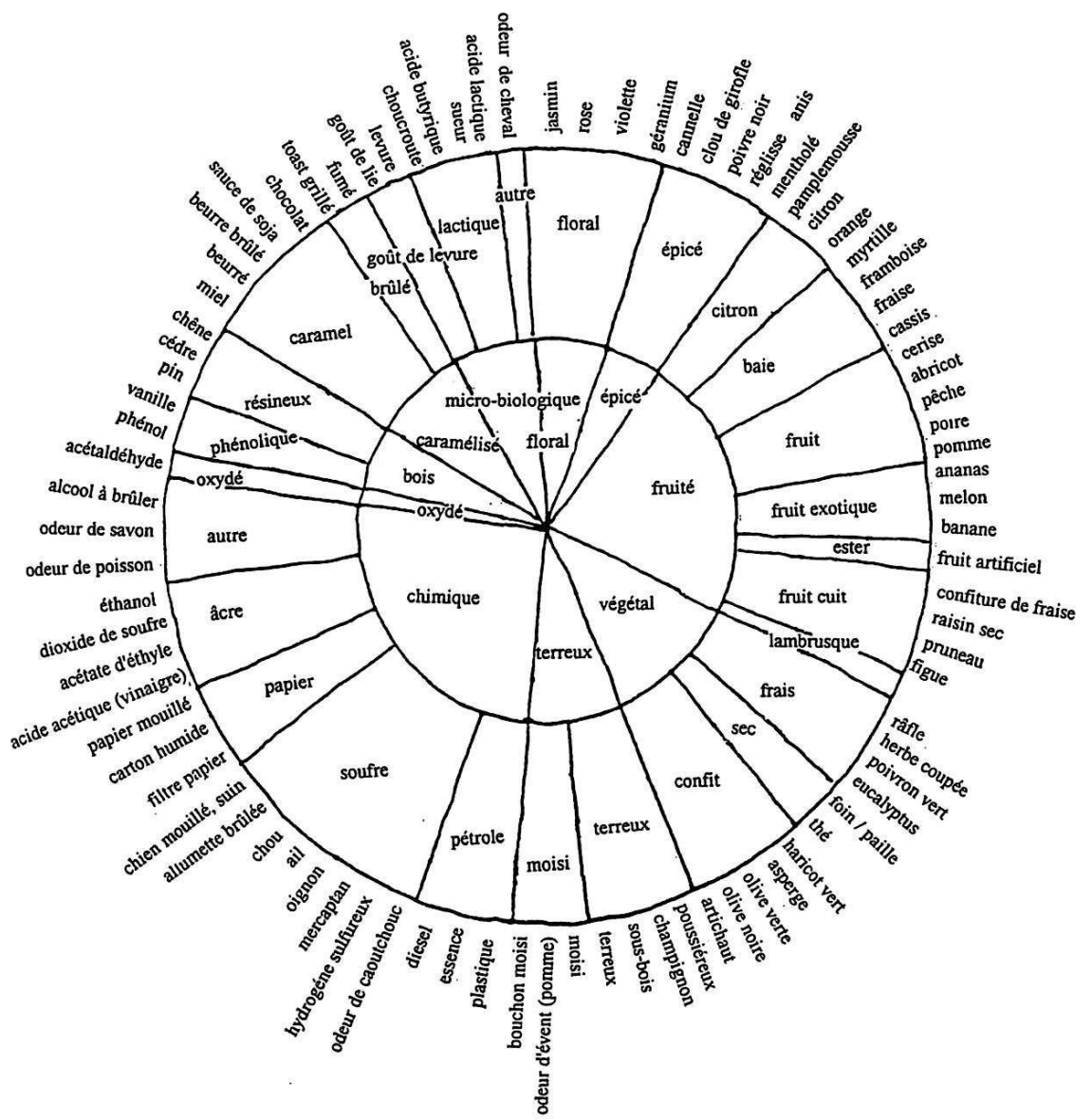

Certains professionnels anglophones emploient avec succès une langue concise mais très évocatrice. De bons exemples sont consultables en ligne : Jancis Robinson (http://www.jancisrobinson.com), Jamie Goode (http://www.wineanorak.com/wineblog), ou, pour une version plus américaine, Joe Roberts (http://www.1winedude.com) ou David White (http://www.terroirist.com). On peut améliorer ses propres compétences par la lecture régulière des fiches d'un dégustateur expérimenté, un peu comme en cuisine le simple fait de feuilleter un livre de recettes peut inspirer l'envie d'en essayer de nouvelles... 


\section{Le défi du vocabulaire}

Pour évoquer les qualités organoleptiques d'un vin, il existe plusieurs groupes de mots, chacun avec ses difficultés particulières.

Ceux qui résistent à la traduction : typicité, ciselé (excellent, celui-là, on le ressent presque sur la langue), ou vineux (un vin qui a le goût de vin !), souvent employé à propos d'un degré d'alcool élevé, difficile à exprimer de façon non négative. D'autres mots posent problème en raison de leur connotation négative en anglais : ainsi, on ne peut parler directement de l'acidité du vin alors même que c'est l'une de ses composantes essentielles. On dira plutôt good acidic balance (bon équilibre de l'acidité) ou acidulous notes (notes acidulées). Sucrosité non plus n'a pas les mêmes implications en anglais et il faut donc trouver le bon équivalent lorsqu'il s'agit de décrire un vin sec.

Gouleyant, gras, aérien, croquant sont également difficiles à traduire. Seule l'approche intuitive permettra d'éviter au texte de "sentir la traduction ". Pour obtenir un résultat le plus naturel possible à la lecture, je procède à une révision bilingue puis unilingue.

Ceux qui ont trop servi : élégant, gourmand, finesse. Rien à leur reprocher sinon une utilisation un peu trop répétée. Si l'auteur emploie plusieurs fois les mêmes mots, je ne m'interdis pas une certaine liberté pour obtenir un texte fluide et agréable à lire en version traduite. D'où la nécessité de disposer d'un bon dictionnaire des synonymes.

Les néologismes : parfois, le mot français semble si parfait qu'en l'absence d'équivalent adéquat, il est anglicisé. C'est le cas du mot minerality, formé pour saisir une expérience quasiment impossible à décrire. Vous ne le trouverez pas dans le dictionnaire et il a fait l'objet de bien des débats avant son acceptation, mais il est désormais largement employé dans le milieu vinicole. La tentation est grande d'en faire autant pour d'autres mots au risque de rendre son texte abscons.

Ceux qui décrivent les défauts du vin : goût de serpillère, de dissolvant, vin réduit (l'opposé de l'oxydation) dont la meilleure solution de traduction est notes of reduction ou reductive aromas. Et puis il y a le mercaptan et son odeur d'œuf pourri. Ces mots, souvent familiers pour l'expert, nécessiteront quelques explications pour le profane.

La spécificité des spiritueux : si le vin est le produit de la fermentation, les spiritueux sont le résultat de la distillation qui a sa terminologie propre : cœur de chauffe, têtes et queues de distillation, la part des anges. Le registre est différent de celui du vin et la force et l'intensité de l'alcool doivent être exprimées de façon positive. Parmi les arômes caractéristiques des spiritueux : tourbé, iodé, salé, fumé, hespéridé, botanique, caramélisé, rancio.

Les termes techniques : à manier avec précaution. Un exemple : " empyreumatique ", qui vient du mot grec empureuma (la braise) et recouvre toute une famille d'arômes aux odeurs brûlées. 
"Désormais, si vous sentez dans votre verre de vin une odeur torréfiée, grillée, fumée, brûlée... vous pouvez lever le petit doigt et lancer d'un ton très dégagé : "Je kiffe bien les nuances empyreumatiques de ce vin". Succès garanti. "

Ophélie Neiman - MISS GLOUGLOU - dans “ M » Le Magazine du journal Le Monde

\section{Conclusion}

La force du traducteur réside dans l'étendue de son expérience autant que dans ses connaissances linguistiques. L'acquisition de savoirs est une source constante de plaisir et d'enrichissement qui demande un esprit ouvert et une curiosité toujours en éveil.

Un traducteur spécialisé doit se tenir en permanence au courant des évolutions dans son domaine. Dans mon cas, cela peut avoir trait à des innovations techniques qui mettent en question la définition du vin, à l'utilisation des ressources naturelles, à de nouvelles techniques œnologiques ou à de nouveaux règlements sanitaires ou normes d'étiquetage. En outre, on ne saurait ignorer la croissance du mouvement écologique avec la multiplication des producteurs passant au bio, les préoccupations pour l'environnement à l'origine de pratiques comme la confusion sexuelle en viticulture et l'intérêt accru pour les vins naturels, donc sans intrants, et le nouveau vocabulaire y afférent.

C'est un monde en constante évolution, ce qui le rend d'autant plus fascinant.

traductionvin@outlook.com

Traduit de l'anglais par Hélène Ladjadj.

Jane Maylin est traductrice depuis plus de 10 ans, spécialisée en viticulture et œnologie. Elle entretient des liens privilégiés avec le monde du vin dans lequel elle a travaillé jadis. En 2013 elle a été évaluée Niveau 1 (excellent) ISO-9001 pour ses traductions. De nationalité anglaise, elle habite la région des Corbières, dans le sud de la France, depuis 1987.

www.traduction-vins-spiritueux.com 